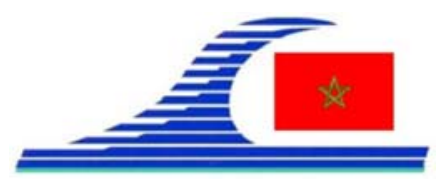

Conférence Méditerranéenne Côtière et Maritime

EDITION 2, TANGER, MAROC (2011)

Coastal and Maritime Mediterranean Conference

Disponible en ligne - http://www.paralia.fr - Available online

\title{
Impact des aménagements portuaires sur l'évolution du trait de côte au niveau du littoral de Sousse Sud
}

\author{
Mongi SOUAYED ${ }^{1,3}$, Ikram BARDI ${ }^{2,3}$, Saadi ABDELJAOUED ${ }^{2,3}$
}

1. Institut Supérieur des Beaux-Arts de Sousse, Place de la Gare 4000 Sousse, Tunisie. mongi_souayed@yahoo.fr

2. Faculté des Sciences de Tunis, Département de Géologie, Tunisie.

3. Laboratoire des Ressources Minérales et Environnement à la Faculté des Sciences de Tunis, Tunisie.

\section{Résumé :}

Le littoral de Sousse Sud (Sousse - Sidi Abdelhamid) est une plage sableuse à pente douce située au sein d'une large baie entre la ville de Sousse au Nord et la Ville de Monastir au sud.

Cette zone côtière subit actuellement un recul du trait de côte très inquiétant. En effet dans certaines parties il a atteint une moyenne d'environ $2 \mathrm{~m}$ par an. Ce littoral est divisé en trois parties, la première protégée par un important enrochement et deux briselames, la deuxième partie assujettie à une érosion importante et à un recul du trait de côte et une troisième partie où l'on assiste à une avancée.

Les processus à l'origine de ce déséquilibre sédimentaire sont liés à l'action dynamique de la houle et des courants littoraux dominants qui font transiter le matériel sableux côtier du Nord vers le Sud. Cette configuration est due à deux principaux facteurs à savoir les aménagements portuaires et l'hydrodynamisme du littoral.

\section{Mots Clés :}

Sousse sud - Côte sableuse - Morphologie côtière - Recul du trait de cote - Avancée du trait de côte - Hydrodynamisme - Dérive littorale

\section{Introduction}

La zone littorale de Sousse Sud (Sousse-Sidi Abdelhamid) est située dans la partie extrême sud du Golfe de Hammamet, précisément entre le port de commerce et de pêche de Sousse et Oued Hamdoun (figure 1). Ce littoral est divisé en trois parties, la première, juste au Sud du port de commerce et de pêche, elle est protégée par un important enrochement et deux brise-lames, elle se termine au niveau de l'Oued Hallouf. La deuxième partie est constituée d'un littoral sableux plat assujetti à une érosion importante et à un recul de la ligne de côte. Cette partie se termine au niveau du marabout de Sidi Abdelhamid. Enfin une troisième partie où l'on assiste à une avancée du trait de côte ; cette partie se termine au niveau de la jetée du port de la centrale thermique juste au Nord de l'Oued Hamdoun. 

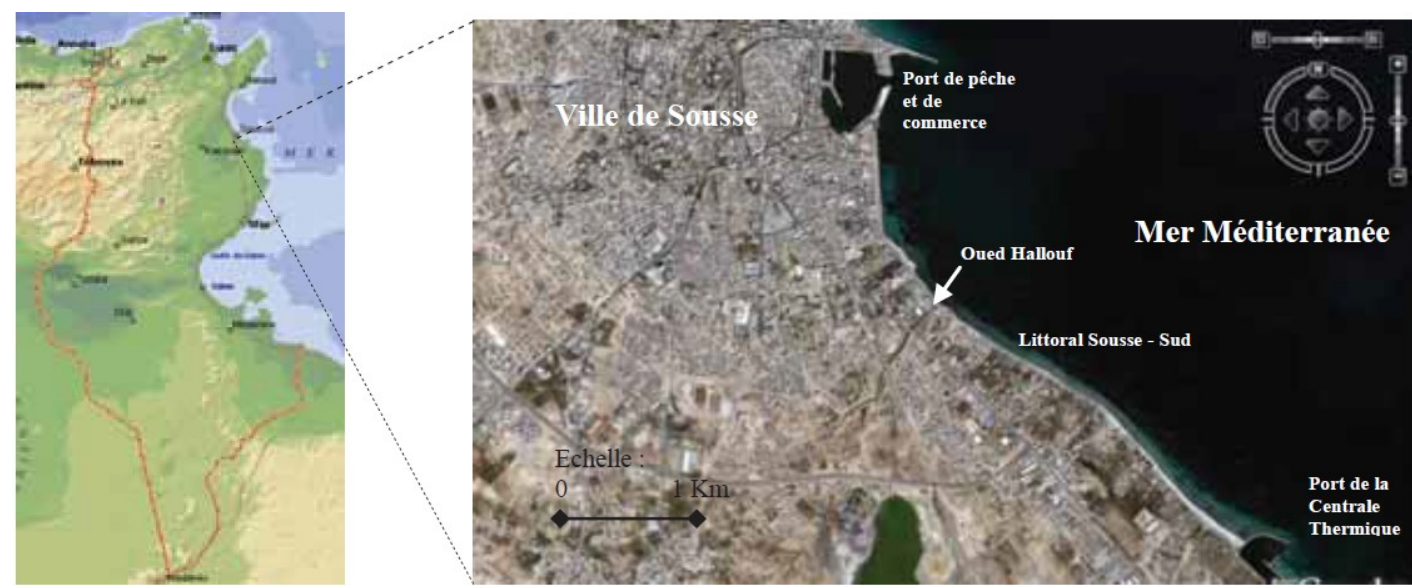

Figure 1. Localisation du littoral de Sousse - Sud (Google Earth, 2010).

\section{Les processus hydrodynamiques}

A la côte, les houles les plus fréquentes proviennent du Nord et du Nord-Est (32\%), de l'Est (27\%) et du Sud-Est (20\%). Mais ceux qui sont à l'origine de la dynamique des courants côtiers sont principalement les vents du Nord à Nord-Est puis les vents du SudEst (STUDI, 1989). Les courants de marée sont très faibles et n’interviennent pas dans la dynamique sédimentaire.

Lors des grandes tempêtes les courants sagittaux n’agissent pas efficacement vu le développement de l'herbier qui augmente avec la profondeur et qui freine à la fois ces courants et aussi les houles. Ainsi le mouvement de sable vers le large est atténué.

Par contre le transit latéral du sable est assez important grâce aux courants littoraux. En effet les vents dominants venant du Nord-Ouest, du Nord et du Nord-Est induisent par réfraction un courant littoral vers le Sud qui se charge du transit du matériel sableux du Nord vers le Sud. L'estimation de ce transit diminué de celui qui s'effectue rarement dans le sens inverse donne une résultante vers le Sud de l'ordre de 10000 à $20000 \mathrm{~m}^{3} \mathrm{an}^{-1}$ de sable (STUDI, 1989).

\section{Evolution du trait de côte entre le port de pêche et le port de la centrale thermique}

Entre le Port de Commerce de Sousse et celui de la centrale thermique, l'évolution du trait de côte de 1962 à 2010 est variable selon les endroits et les aménagements qui y ont été réalisés. On distingue ainsi trois parties :

- Dans la première juste au Sud du Port de Commerce et de pêche (figure 2), la côte a évolué dans le temps et dans l'espace à la suite de différentes interventions anthropiques. De 1962 à 1983 on avait assisté à un recul de la ligne de côte qui avait menacé la barrière qui protège l'avenue Hédi Nouira. De 1983 à 1996 on avait assisté à une avancée du trait de côte après la réalisation des deux brise-lames. Actuellement 
cette avancée s'est arrêtée au niveau des deux brise-lames à $50 \mathrm{~m}$ de la barrière initiale.

- La deuxième partie sableuse (figures 3 et 4) a été marquée pendant une première période de 1963 à 1986 par un certain équilibre et une stabilité du trait de côte, la deuxième période de 1986 et se poursuit jusqu'à présent, par un recul du trait de côte atteignant à certains endroits plus de $2 \mathrm{~m}$ par an.

- Enfin la troisième partie où l'on assiste à une avancée du trait de côte de l'ordre de 2 à $3 \mathrm{~m}$ par an; cette partie se termine au niveau de la jetée du port de la centrale thermique juste au Nord de l'Oued Hamdoun.

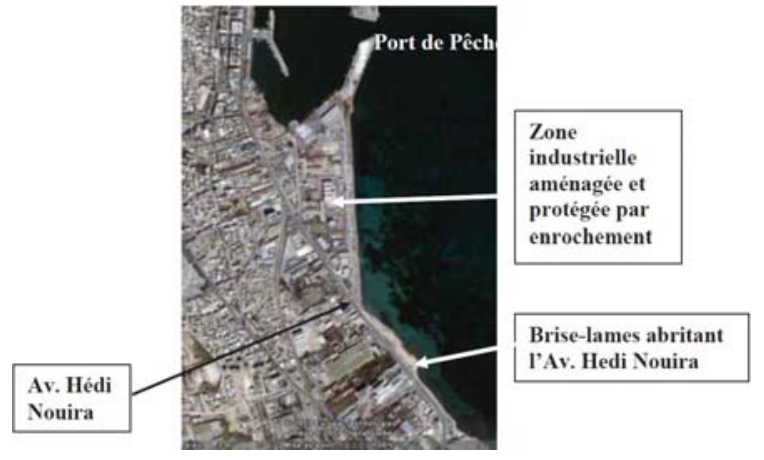

Figure 2. Côte aménagée au Sud du port de pêche de Sousse (d'après image satellite Google Earth, 2007).

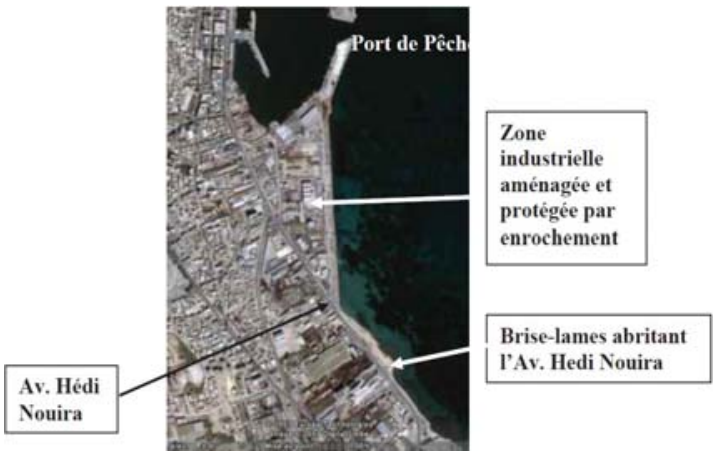

Figure 3. Etat du littoral de Gaïed Souassi en 1963.

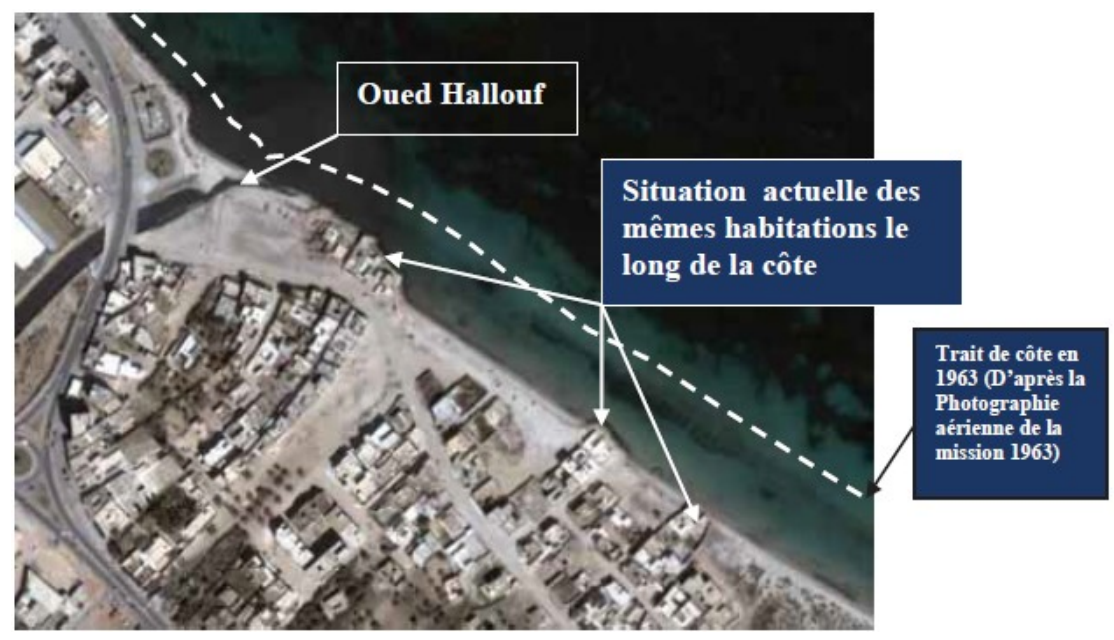

Figure 4. Etat du littoral de Gaïed Souassi en 2010

(d'après image satellite Google Earth, 2010). 
La connaissance de la Mer :

un vecteur du développement durable en Méditerranée

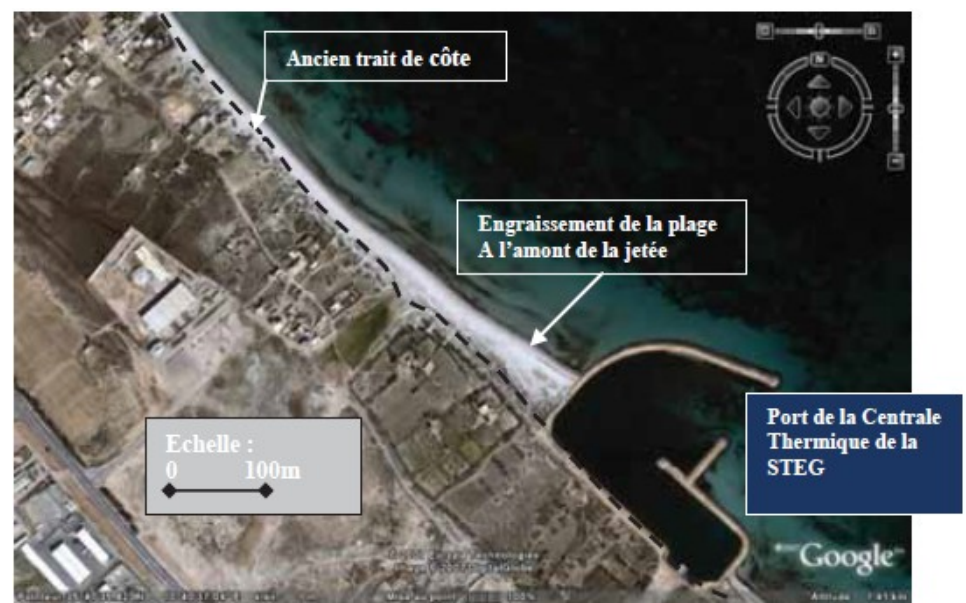

Figure 5. Etat du littoral à l'extrémité Sud au niveau de la centrale thermique en 2003 (d'après image satellite Google Earth, 2010).

\section{Conclusion}

A partir des observations sur le terrain, il est évident que le recul de la ligne de côte sur le littoral de Sousse Sud est le résultat d'un déséquilibre sédimentaire lié à l'action dynamique de la houle et des courants littoraux accentués par l'intervention humaine. Les courants littoraux dominants se chargent de faire transiter le matériel sableux côtier du Nord vers le Sud.

Cette érosion constitue une menace non seulement pour les habitations mais aussi pour l'environnement. En effet par endroits la ligne de côte recule en moyenne de plus de $2 \mathrm{~m}$ par an, et les habitations qui sont actuellement soumises au front des vagues (figure 4) risqueront de se trouver en pleine mer si une solution n'est pas trouvée le plutôt possible. Certaines d'entre elles y sont déjà, d'autres sont en partie détruites.

\section{Références bibliographiques}

STUDI (1989). Etude de dix ports de pêche côtiers. Rapport général sur les observations hydrologiques et climatiques. Rapport interne D.G.S.A.M. Ministère de l’Equipement. 